

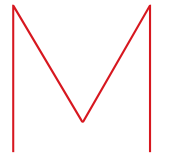

other Nature is a lazy chemist. Occasionally, she produces an organic molecule that's a winning drug. But more often, a nature-made chemical's medicinal powers are coupled with flaws, such as brutal side effects. Until recently, upping the safety of those drugs by retooling their parts was a lot like assembling Ikea furniture.

Take amphotericin B, a lifesaving but toxic antifungal medicine. To build a better version, chemists would need to translate a series of tauntingly simple stick-figure diagrams into actual chemistry, coming up with chemical reactions to attach and rejigger dozens of atomic parts. This hands-on approach could take more than 100 steps.

Enter automation. A new technique can snap a potential new drug together in just a few steps. The system is like chemistry's Easy-Bake oven: assembling premade ingredients and serving up custom small molecules at the push of a button.

It's just one of several high-tech ways chemists are beginning to make new molecules in record time. One setup performs classic chemical reactions with programmable robotic arms, shakers and other gizmos. Another builds molecules by unleashing streams of chemical cocktails through dizzying obstacle courses of hoses, pumps and valves.

"If we can deliver on-demand small molecule synthesis to the world, this would be absolutely transformative," says chemist Martin Burke of the University of Illinois at UrbanaChampaign, who recently unveiled his prototype. Those ondemand molecules could be the next lifesaving drugs or new materials for reaping solar energy.

Yet many chemists are reluctant to embrace such technologies. Some say it's time they catch up. "The automotive industry did it in the '70s," says biochemist Andrew Mesecar of Purdue University in West Lafayette, Ind., alluding to Detroit's machine- and robot-enhanced assembly lines. "Chemistry," he says, "is lagging behind."

Doubt among chemists that automation will be truly useful is a factor, Mesecar says. Plus, there's lingering skepticism and bitterness over past machine-based efforts. In the 1990s, technologies capable of gushing hundreds of arbitrarily generated molecules promised an end to tedious, painstaking, step-by-step synthesis. But, after investing time and money, most chemists who fished through the resulting deluge of compounds came up empty-handed.

Those early tech backers did themselves in by overpromising, says medicinal chemist Craig Lindsley of Vanderbilt University Medical Center in Nashville.

But a small band of researchers, including Burke and Mesecar, never gave up on automation. Instead of mass producing a flood of random molecules, these researchers turned to automated systems that make artisanal molecules - fast and cheap. Learning from the past, the new automation champions

This mess of tubing and pumps snaps together molecules from chemical building blocks. Developer Martin Burke imagines the automated system freeing up chemists' time to dream up the next blockbuster drug. pitch the technology as a useful tool, rather than a cure-all.

"It's not been easy," says synthetic chemist Steven Ley of the University of Cambridge. Minus the hype, more scientists are now open to what modern, technology-assisted chemistry could look like. "What you're seeing is a sea change."

\section{Snappy charm}

Chemists are accustomed to crafting molecules through dozens of arduous steps that might yield a blockbuster drug, but more often result in pharmaceutical flops. On white boards and scraps of paper, researchers sketch hexagons and zigzags, the backbones of molecules. By drawing an additional oxygen atom on one side of a molecule or squeezing a nitrogen group onto the other, for example, chemists try to subtly change the design to make better molecules. Maybe those improvements will knock out a drug's side effects or beef up a material's toughness. But once they are moved from the drawing board into round-bottom flasks, forging and sculpting those modified molecules can take years of trial and error.

Burke was eager to move more quickly. In the late 1990s, he realized that the antifungal amphotericin $\mathrm{B}$, also known as AmB, needed tweaking. The potent drug can wipe out systemic fungal infections in critically ill patients. But it has severe side effects, including kidney damage. He wanted to make a safer version without delay.

He started with a well-known chemical trick and then developed one of his own to make the chemistry hands-free. The old flask-based chemistry scheme allowed him to snap fragments of molecules together like Lego building blocks. The method works by forging carbon-carbon bonds between molecular fragments. Each block has a linker. In a chemical reaction, the linkers help bring the two molecular fragments together and connect their carbons.

By adding a compound with a halogen (elements such as bromine and chlorine) as a linker on one side of a molecular fragment and a boronic acid on the other, the building blocks can snap together like train cars. But with two essentially sticky ends on each block there was no way to control which ends were coupling and in what order.

Clever caps Using a chemical cap called MIDA, researchers can control the assembly of potential drugs using linkable molecular fragments, shown here as blocks. Each block has a boronic acid linker on one end and a halogen-containing linker on the other. With the removable MIDA cap covering the boronic acid, the blocks can snap together in only one way (left). With no cap, the blocks have two options (right), which is problematic. SOURCE: J. LI ET AL/SCIENCE 2015

\section{Building a small molecule}

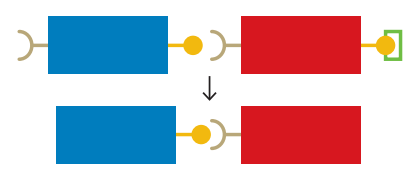

$\downarrow$

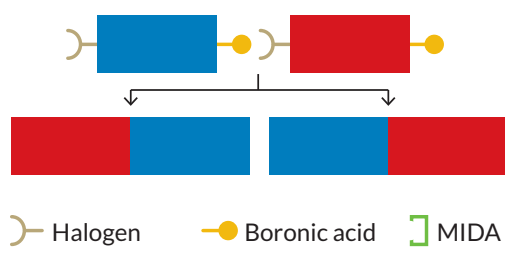




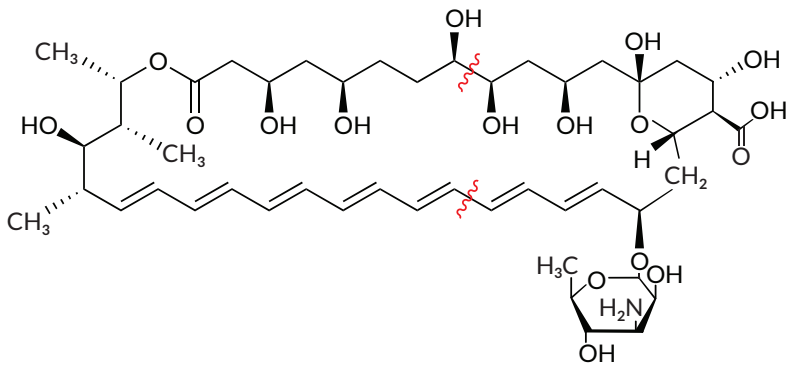

$\mathrm{AmB}$

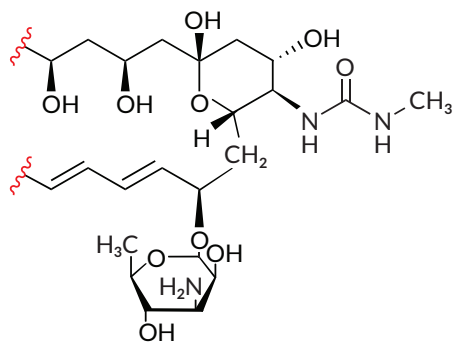

AmBMU

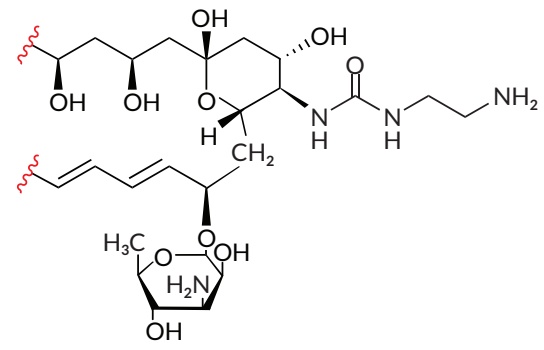

AmBAU
In 2007 in the Journal of the American Chemical Society, Burke and Eric Gillis, now at Bristol-Myers Squibb, reported their trick to control coupling: a removable cap, called MIDA ( $N$-methyliminodiacetic acid), that fits over the boronic acid to keep it from hooking up until desired.

With around 200 building blocks already commercially available for snappy assembly - and more coming - the team estimates that their method could quickly make thousands of druglike compounds. The technique also allows researchers to make tiny adjustments to existing drugs, including AmB. Earlier this year, Burke and colleagues reported in Nature Chemical Biology that, using a mix of automated and traditional methods, they created versions of AmB that have lesssevere side effects in mice.

The cap also paved the way for machines to take a larger role. Burke's team designed an automated apparatus that links the building blocks, purifies coupled blocks, strips MIDA caps off new blocks and then continues linking. The researchers revealed their synthesizer in a paper published in Science in March.

The chemistry and the machine are "really cool," says organic chemist Damian Young of Baylor College of Medicine in Houston. Carbon-carbon bonds are one of the harder bonds to make. They're the reason some synthetic methods remain "a bit of a black box," he says, keeping custom molecules out of reach for some researchers.

But Burke's method has limitations. Users will be restricted by the types of building blocks available and the reaction

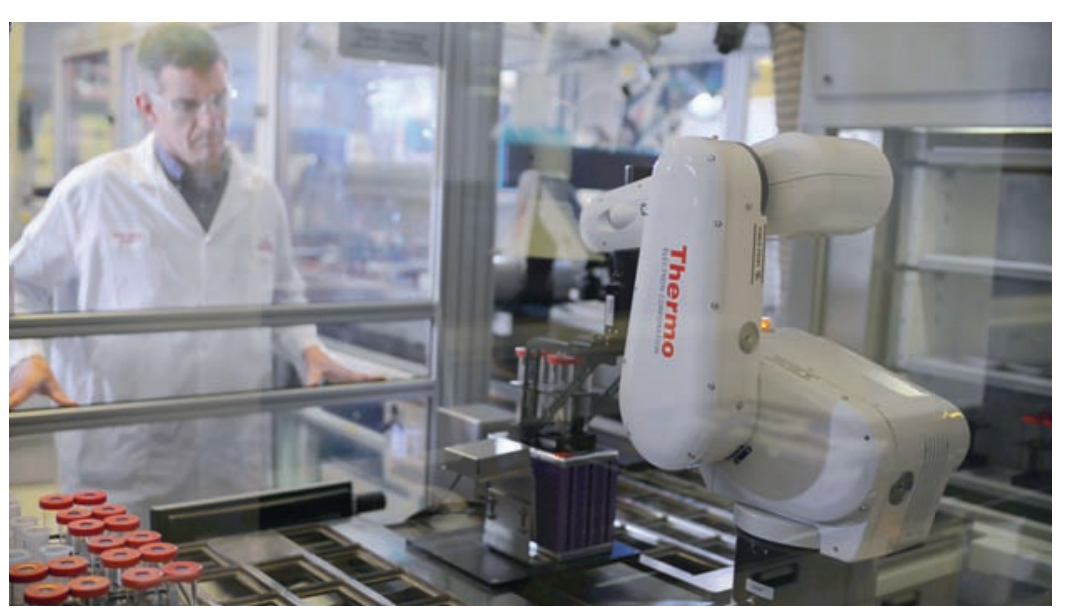

Helpful tweaks The antifungal medication amphotericin B, or $\mathrm{AmB}$ (left), comes with dangerous side effects, but has been difficult to improve using traditional chemical methods. Some new tricks allow researchers to quickly and easily modify the drug's structure. That work guided the design of two new AmB variants (above), both better at specifically targeting fungus, thus reducing toxicity in mice. SOURCE: S.A. DAVIS ET AL/NATURE CHEMICAL BIOLOGY 2015

conditions, such as the nature of the solvent, that the machine can handle, says Amy Ripka. She is executive director of medicinal chemistry at WuXi AppTec, a drug and medical device company based in Shanghai.

Ripka is generally skeptical of automated chemistry. "I think it's like the housing bubble," she says. "People get all excited about these new technologies." Then, she says, the technology doesn't solve all problems and optimism bursts.

Lindsley acknowledges the concerns and reiterates the caveats of automation proponents: It's more a tool in an arsenal than a panacea.

\section{Tubes and time machines}

One system is already proving that automation can be a crucial time-saver, according to Mesecar and his colleague, Purdue organic chemist Sarah St. John. The pair partnered with pharmaceutical giant Eli Lilly, based in Indianapolis, to use the company's fully robotic, remote-controlled chemistry lab, the Automated Synthesis Lab. Without a human in sight, the lab can run dozens of standard chemical reactions, popping oxygen or nitrogen atoms onto molecules as needed.

Mesecar and St. John are making small molecules to cripple coronaviruses, a family of viruses that can cause respiratory diseases such as SARS and MERS. Like Burke's work with AmB, the researchers had a starting point: molecular structures that they wanted to tweak. Their molecules attacked a vital coronavirus enzyme but were too weak to stop the virus.

From her university lab, St. John remotely programmed all of the chemical steps needed to modify the molecule and yield more potent assailants. At Lilly's automated lab a couple

More than 200 researchers worldwide have made chemical compounds using a fully automated, remote-controlled synthesis lab at Eli Lilly in Indianapolis. 
of towns away, robotic arms swung into action, starting reactions, adding chemical ingredients and ultimately creating around 50 molecules within a month. It took a lot of front-end programming, St. John says. But it paid off. Six of the compounds can hamper the enzyme in all 10 coronaviruses that she has tested. A few other molecules foil the enzyme of one virus.

With her time freed up to do other experiments, St. John estimates the robotic help saved her three to six months of work.

Few researchers, however, have this kind of help. Fully robotic labs, such as Eli Lilly's, are expensive to build and therefore rare outside of well-funded pharmaceutical companies.

Some scientists think they can do better anyway. Robotic help is too much like an automotive assembly line: not modern enough. Says Ley, of Cambridge, "It's a little boring and a little bit conservative."

Ley and others are trying to develop cheaper machines that can also do more challenging chemistry feats. He's particularly focused on flow chemistry, which carries out reactions as solutions stream through tubes and swirl together to make new compounds. The fluid system allows chemists to set up a chain of chemical reactions that progress without assistance as the chemicals gush through the pipes. The tightly controlled series of reactions can be safer than conventional methods, avoiding vats of unpredictable reactions.

New gadgets and technologies can plug into the labyrinth of piping to carefully control and monitor reactions. In January, Ley and colleagues described a flow system that performed seven steps in one go to produce a druglike molecule. That work was reported in Angewandte Chemie International Edition.

What's most exciting, he says, is just bringing more tech to the bench. Ley belongs to a network called "Dial-a-Molecule" that shares this aim. Spearheaded by organic chemist Richard Whitby of the University of Southampton, the group's ultimate goal is to develop powerful machines to quickly and automatically design and create any molecule a chemist could think up. One specific emphasis of the group is to design software that can predict which chemical reactions will work and even come up with step-by-step assembly plans. It would remove the need for much human trial and error.

Last year, Ley and colleagues reported in Beilstein Journal of
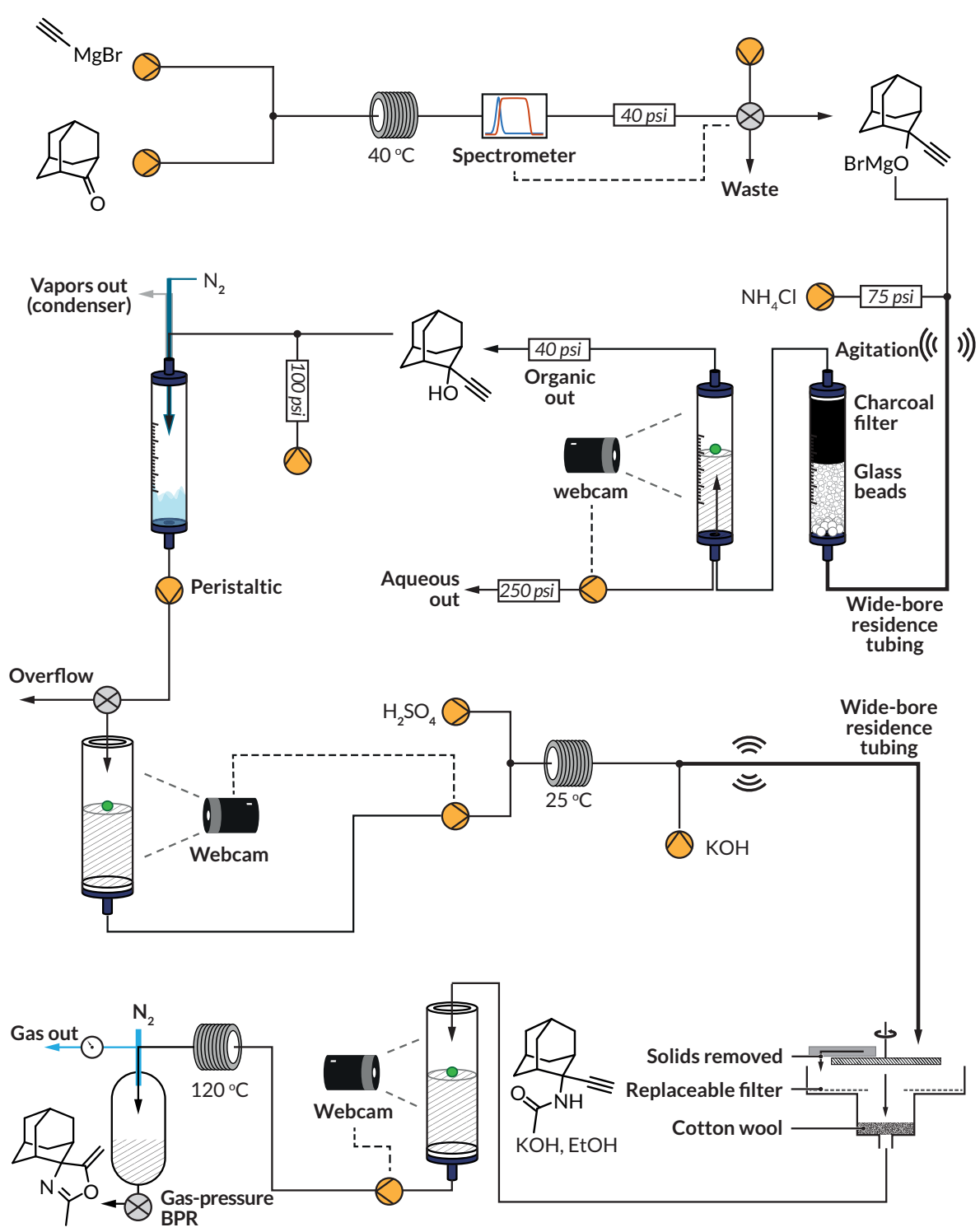

Swoosh Researchers are building molecules by streaming chemicals through networks of tubes, pumps and columns. The method, called flow synthesis, runs a series of automated reactions as the chemicals spurt through an obstacle course-like setup (one outlined above). By plugging computers and cameras into the network, researchers can control and monitor the progress of reactions.

Organic Chemistry how to program a cheap, simple computer called Raspberry Pi to run networks of chemical equipment, monitor complex reactions and upload data to the Internet.

"We could take a huge step forward," Whitby says of the growing effort to add more tech to the lab. Any automation naysayers are going to be left in the dust, he predicts. To which he adds: "I have no sympathy."

\section{Explore more}

- Junqi Li et al. "Synthesis of many different types of organic small molecules using one automated process." Science. March 13, 2015. 